\title{
Hesitancy Towards COVID-19 Vaccine Among Older People in Luzhou, China: A Cross-sectional Study
}

\author{
Jianlan Ren ( $\nabla$ 370319003@qq.com ) \\ the Affiliated Hospital of Southwest Medical University \\ Yu Zheng \\ the Affiliated Hospital of Southwest Medical University \\ Yue Luo \\ Southwest Medical University \\ Mei Li \\ Southwest Medical University

\section{Rendie Xie} \\ the Affiliated Hospital of Southwest Medical University

\section{Daiying Zhang} \\ the Affiliated Hospital of Southwest Medical University
}

\section{Research Article}

Keywords: Vaccine Hesitancy, COVID-19, Acceptance, The elderly

Posted Date: August 24th, 2021

DOI: https://doi.org/10.21203/rs.3.rs-757239/v1

License: (c) (1) This work is licensed under a Creative Commons Attribution 4.0 International License.

Read Full License 


\section{Abstract}

Background:Vaccination is one of the most effective and low-cost health measures to prevent COVID-19. Vaccine hesitancy is an obstacle to COVID-19 vaccination.

Objective:This study aims to inform the vaccine hesitancy and analyze related factors towards COVID-19 vaccination among elderly in Luzhou, so as to provide suggestions for increasing vaccines uptake.

Methods:A face-to-face questionnaire cross-sectional survey was conducted among the elderly people over 60 years old in Luzhou City in April 2021 by multi-stage sampling method.

Results: A total of 1047 valid questionnaires were received. $42.0 \% \varangle 440 / 1047 \rrbracket$ vaccine hesitancy was reported among elderly in total. Female (OR=1.282, 95\%Cl:1.003-1.649), live in the rural (OR=3.659, $95 \% \mathrm{Cl}: 2.813-4.758)$, poor health $(\mathrm{OR}=2.318,95 \% \mathrm{Cl}: 1.473-3.649)$, individuals not having regular medical check-ups (OR=2.669, 95\% Cl:2.072-3.437) and no history of self-funded influenza vaccination ( $\mathrm{OR}=2.408,95 \% \mathrm{Cl}: 1.656-3.502)$ reported slightly higher vaccine hesitancy. Being in low-risk areas and no need to get vaccinated $(\mathrm{OR}=16.877,95 \% \mathrm{Cl}: 11.750-24.242)$, being considered that the current protective measures had been able to avoid infection ( $\mathrm{OR}=5.539,95 \% \mathrm{Cl}: 3.596-8.532)$, being considered that the natural immunity get from the disease was better than getting COVID-19 vaccine (OR=5.297, 95\%Cl:3.666-7.653), being concerned about side effects (OR=5.155, 95\% $\mathrm{Cl}: 3.655-7.270)$ and being concerned that the vaccine was ineffective $(\mathrm{OR}=10.244,95 \% \mathrm{Cl}: 6.831-15.362)$ had higher prevalence of vaccine hesitancy. Those who believing the COVID-19 vaccine was safe and reliable (OR $=0.075$, $95 \% \mathrm{Cl}: 0.044-0.130)$, the vaccine was free $(\mathrm{OR}=0.466,95 \% \mathrm{Cl}: 0.312-0.697)$ and individuals with higher education ( $\mathrm{OR}=0.189,95 \% \mathrm{Cl}: 0.126-0.284)$ were more willing to get vaccinated.

Conclusion: Confidence in vaccines and perceptions of benefits and risk are associated with vaccine hesitancy. It's very important to strengthen vaccine health literacy education for older people and enhance vaccine confidence. Countries need to supervise the public opinions in social media, television broadcasting and other media, so as to ensure the correct orientation of public opinion. Open and transparent evidence-based information is also needed which can help improve the vaccination coverage rate of the public.

\section{Introduction}

The global COVID-19 pandemic is still continuing, making a great difference to economy, politics and culture. Through strict and effective public health emergency control measures, China has achieved great success in controlling COVID-19. Widespread COVID-19 vaccination is essential to stem the epidemic of COVID-19. ${ }^{1}$ In order to control the pandemic of COVID-19, many countries including China has been actively developing vaccines since COVID-19 occurred. China officially launched an emergency notification for the use of COVID-19 vaccine on July 22, 2020. Up to now, COVID-19 vaccination has been carrying out orderly in many countries around the world, but vaccine hesitancy has become an obstacle to achieving herd immunity. 
Vaccine hesitancy can lead to lower vaccination rates, which in turn affects population immunity. Vaccine hesitancy is influenced by many factors, which is listed as one of the top 10 threats to global public health by the World Health Organization. ${ }^{2}$ In 2012, the WHO's Strategic Advisory Group of Experts (SAGE) identified vaccine hesitancy as the refusal or delay of a vaccine when vaccine services are available. ${ }^{3}$ Confidence, complacency and convenience ("3Cs" model) proposed by the SAGE Working Group are important influencing factors of vaccine hesitancy. Vaccine confidence is a global public health issue. ${ }^{4}$ One study found that more confidence and less complacency were associated with stronger COVID-19 vaccination intention. ${ }^{5}$ The study also pointed out that complacency (a perception of a low risk of the disease) was associated with influenza vaccine vaccination. Furthermore, vaccination convenience (e.g., availability, affordability and geographical accessibility of vaccines so on) is also an important factor. ${ }^{3}$

According to China's official data, up to May 1, 2021, China reported that a total of 270.406 million doses of COVID-19 vaccine were taken. However, due to the large population base in China, the uptake rate of COVID-19 vaccine is still very low, far from the coverage needed to achieve herd immunity. Because of the weak body resistance, and often accompanied by some basic diseases, the elderly infected with COVID19 are usually more serious and more complications.So in China, they are the high-risk population of COVID-19 infection and the key population for prevention and control. Given the importance of vaccination among old people, it is important to understand their attitudes towards COVID-19 vaccination. This study aims to understand the vaccine hesitancy and related factors among old people through a cross-sectional survey, so as to provide information and recommendations for COVID-19 vaccination activities.

\section{Material And Methods}

\section{Study Design and Population}

A cross-sectional survey was conducted among old people in Luzhou, China. The method of multi-stage sampling was adopted to select 4 districts in Luzhou, and 3-4 communities were selected from each district. The convenient sampling was used to conduct face-to-face questionnaire survey among the elderly who met the survey conditions in the communities. Inclusion criteria: the permanent resident population of Luzhou ( $\geq 6$ months), aged 60 years old at the time of the survey, volunteer to participate in this study and give informed consent, normal thinking, certain listening, speaking and understanding ability, and no mental illness or consciousness disturbance .

\section{Measure}

We compiled a questionnaire based on the $3 \mathrm{C}$ model of vaccine hesitancy. The questionnaire included basic demographic information (e.g., gender, age, region, marital status, profession, physical health, education and regular physical examination.), access to epidemic information, vaccination intention, confidence, complacency, and accessibility and convenience of COVID-19 vaccination services. We 
invited six experts working in infectious disease research and public health to conduct questionnaire consultation. After two rounds of expert consultation, the final questionnaire was formed. The scale level content validity index, S-CVI/Ave was 1.00 based on the six experts in China. We conducted a pre-survey among 54 elderly people aged $\geq 60$ years old, and 54 valid questionnaires were collected. All study subjects reported that the questionnaire items were easy to understand and the survey method was acceptable. Based on the pre-survey data, the reliability of the questionnaire was analyzed. The main contents of the questionnaire (confidence, complacency and service convenience and accessible of COVID-19 vaccine) were assessed by 5-point Likert scale. Five-point Likert scale (strongly disagree, agree, unsure, agree, strongly agree) were divided into three categories: disagree, unsure, and agree. The Cronbach's a coefficient of the questionnaire is 0.806 which is a 'reliable' level. ${ }^{6}$

To measure vaccine hesitancy, participants were firstly asked "Have you been vaccinated against COVID19?". The answers included "Yes and No". Those who had not been vaccinated were asked again "Would you like to take the COVID-19 vaccine if it is available now?". The response options included "Yes, No and Undecided". The population was divided into two groups ("Vaccine Hesitancy" and "Not Vaccine hesitancy"). With reference to the definition of vaccine hesitancy and some related research, ${ }^{3,7-9}$ we define "Vaccine Hesitancy" as people who were undecided and unwilling to get vaccinated. Those who had been vaccinated and would like to be vaccinated were classified as "Not Vaccine Hesitancy".

\section{Data Collection}

The questionnaires were collected from April 10 to April 30, 2021. The seven investigators involved in this study have all received unified training and have good communication skills. With the consent of the participants, they were given one-on-one questionnaires by full-time investigators. After answering the questions, the questionnaires were collected.

\section{Ethical Consideration}

Based on the principle of anonymity and informed consent of the participants, data was collected. This study was approved by the Affiliated Hospital of Southwest Medical University (No: KY2021075).

\section{Statistical Analysis}

Epidata 3.1 was used to establish the database and the logic verification was carried out to ensure the accuracy of the data input. SPSS 21.0 was used for statistical analysis. The influencing factors for vaccine hesitancy were firstly identified by univariate analysis, and then the multivariate analysis was performed by binary logistic regression. $\mathrm{P}<0.05$ indicates statistically significant.

\section{Results}

A total of 1047 elderly with an average age of $(67.65 \pm 6.397)$ years were investigated. $51.1 \%$ participants were males. Urban elderly accounted for $62.4 \%$, and rural elderly accounted for $37.6 \%$. Primary education and below, junior and senior high school education,college education accounted for $28.0 \%, 54.3 \%, 17.8 \%$ 
respectively. Unmarried, married, divorced, widowed accounted for $1.9 \%, 86.7 \%, 2.3 \%, 9.1 \%$ respectively. Older people were mainly from farming (30.4\%) and government and public institutions $(25.0 \%) .60 .0 \%$ of the older people considered their health to be average, and 55.7 percent of them attended regular physical check-ups.84.2\% of the elderly had not been vaccinated against influenza at their own expense.There were diverse ways to obtain information about COVID-19 vaccine, including mainly television broadcasting (56.3\%), Relatives, neighbors and friends (50.0\%), and WeChat, QQ and other social networks(34.2\%).The demographic characteristics of the participants are shown in Table 1.

One hundred and seventeen older people (11.2\%) had taken the COVID-19 vaccine, which were divided into "Not Vaccine Hesitancy". Among those who did not receive the COVID-19 vaccine $(n=930)$, the number of older people would like to be vaccinated against COVID-19 was 490 , accounting for $52.7 \%$. Therefore, the total number of "Not Vaccine hesitancy" was 607, accounting for $58.0 \%$ among all older people. Those reporting that they were unwilling to get vaccinated $(n=121,11.6 \%)$ or undecided to take the COVID-19 vaccine $(n=319,30.5 \%)$ were classified as vaccine hesitancy, which reported in 440 of 1047 in total (accounting for $42.0 \%$ ).

The results of Binary logistic regression are shown in table 2. Female $(\mathrm{OR}=1.282,95 \% \mathrm{Cl}: 1.003-1.649)$, living in the rural $(\mathrm{OR}=3.659,95 \% \mathrm{Cl}: 2.813-4.758)$, poor health $(\mathrm{OR}=2.318,95 \% \mathrm{Cl}: 1.473-3.649)$, individuals not having regular medical check-ups $(\mathrm{OR}=2.669,95 \% \mathrm{Cl}: 2.072-3.437)$ and no history of selffunded influenza vaccination ( $\mathrm{OR}=2.408,95 \% \mathrm{Cl}: 1.656-3.502$ ) was a risk factor for vaccine hesitancy. In the "3Cs" model, Being in low-risk areas and no need to get vaccinated (OR $=16.877,95 \% \mathrm{Cl}: 11.750$ $24.242)$, being considered that the current protective measures had been able to avoid infection ( $O R=$ $5.539,95 \% \mathrm{Cl}: 3.596-8.532)$, being considered that the natural immunity get from the disease was better than getting COVID-19 vaccine $(\mathrm{OR}=5.297,95 \% \mathrm{Cl}: 3.666-7.653)$, being concerned about side effects of the vaccine $(\mathrm{OR}=5.155,95 \% \mathrm{Cl}: 3.655-7.270)$ and being concerned that the vaccine was ineffective (OR= $10.244,95 \% \mathrm{Cl}: 6.831-15.362)$ were risk factor for vaccine hesitancy, indicating that older people with these statements had more rejection or unsureness towards COVID-19 vaccination. Those people who believing the COVID-19 vaccine was safe and reliable ( $\mathrm{OR}=0.075,95 \% \mathrm{Cl}: 0.044-0.130)$, when the vaccine was provided for free $(\mathrm{OR}=0.466,95 \% \mathrm{Cl}: 0.312-0.697)$, and individuals with higher education $(\mathrm{OR}=$ $0.189,95 \% \mathrm{Cl}: 0.126-0.284)$ had more likeliness to receive the COVID-19 vaccine.

\section{Discussion}

Herd immunity through vaccination to control COVID-19 pandemic requires adequate vaccination coverage, but vaccine hesitancy made universal uptake of COVID-19 vaccines a challenge. In this study, about $58.0 \%$ of older people were willing to get vaccinated against COVID-19, which is significantly lower than the previously study ${ }^{10-12}$ that reported approximately $90 \%$ of the general population in China and about $84 \%$ of the elderly in Shanghai would be willing to be vaccinated. Wang et al. ${ }^{10}$ conducted a survey in March 2020 and found that there was no significant difference in age and region of vaccination uptake. In that study, females reported slightly higher vaccine hesitancy $(41.4 \%)$ than males. This may be because males were more likely to resort to pharmaceutical methods while females preferred to adopt 
non-pharmaceutical behaviors compare with males, such as, hand washing, masking and sanitation. ${ }^{13}$ Another study had the same finding that males are more willing to have the COVID-19 vaccine. ${ }^{14}$ This may indicate that females are more likely to express concerns about the vaccine's effectiveness and safety. According to a study, Older people in Canada, Poland, France, Germany, Sweden, and the UK were significantly more favorably disposed to vaccination than younger respondents, but the reverse trend held in China. ${ }^{12}$ This indicates that the degree of vaccine hesitancy in the older people is not low, which will be a huge challenge to achieve herd immunity.

Meanwhile, older people are more likely to to be infected with COVID-19 due to their body's resistance. Therefore, it is crucial to take effective evidence-based measures to reduce vaccine hesitancy among older people.

We found that most factors for vaccine hesitancy among different population, as well as the older people in our study, were associated with concerns about the effectiveness and safety of vaccines. ${ }^{15}$ In this study, lack of confidence in the COVID-19 vaccine (believing the vaccine was ineffective and being afraid of side effects) was a risk factor for vaccine hesitancy, consistent with multiple studies. ${ }^{16-19}$ The public may be hesitant about vaccine when there was insufficient information and data on the safety and effectiveness of the vaccine, and they would prefer to wait for reviewing more data to help them make vaccination decisions. ${ }^{20}$ One study pointed out that while more than $90 \%$ of participants would be willing to receive the COVID- 19 vaccine, about $50 \%$ said they would postpone the vaccination until the safety of the vaccine was confirmed. ${ }^{10}$ The effectiveness of a new vaccine is an important indicator of its effectiveness and efficacy. A cross-sectional study found that the public's acceptance rate of the $95 \%$ effective COVID-19 vaccine was $93.3 \%$, but acceptance rate of the $50 \%$ effective vaccine dropped to $67 \%{ }^{21}$ This suggests that the cognition of the benefits and risks of vaccines has a great influence on vaccination decision-making behavior. The public would weigh the risks and benefits of vaccination against available vaccines information and data to make the most appropriate decision for themselves. Therefore, It is necessary for governments to adopt targeted strategies to strengthen public trust in vaccines by using scientific evidence and data to explain the possible benefits and risks of COVID-19 vaccination.

China is implementing free vaccination of the COVID-19 vaccines for all citizens. Through our survey, we found that free vaccines policy increased the willingness of older people to get vaccinated. This may suggest that older people are concerned about cost when deciding to get vaccinated. Compared with free vaccination, higher out-of-pocket costs may have a large impact on vaccination coverage. An early study in China found that public was more willing to accept COVID-19 vaccine when the vaccine was free available. ${ }^{22}$ Therefore, in order to solve the availability of vaccine service, the country should consider the people's ability and willingness to pay when formulating vaccine charging standards.

The study has some limitations. Firstly, the intention of taking COVID-19 vaccine is affected by many factors. The intentions and attitudes of vaccination are not the actual vaccination behavior and may 
change with environment, time, information and other factors. Secondly, due to the limitation of sampling method, the limited representativeness of the sample may affect the universality and generalization of the research results. Therefore, follow-up studies should be continuously improved to deepen the relevant research on the elderly's COVID-19 vaccine hesitancy.

\section{Conclusions}

The older people in Luzhou have higher hesitation about COVID-19 vaccine. We have confirmed that concerns about safety and efficacy of vaccines are barriers to get vaccinated. And individual perceived risk is a positive factor for vaccination. The accessibility of vaccine services, such as providing free vaccines is an important booster for vaccination. In the context of the COVID-19 global pandemic, it is necessary to actively carry out targeted publicity and strengthen vaccine health literacy education among the older people, which has a potential role in improving vaccination coverage. As social platforms and the news media are important sources of vaccination information, strengthening the management of information and news on vaccines is also critical to improving public confidence in vaccines.

\section{Declarations}

\section{Author Contributions Ethics approval and consent to participate}

This study was approved by the Ethics Committee of the Affiliated Hospital of Southwest Medical University, Luzhou.

Based on the principle of informed consent, this study was anonymous and voluntary.

\section{Consent for publication}

All authors read and approved the final manuscript for publication.

\section{Availability of data and material}

All data is included in the manuscript.

\section{Competing interests}

No conflict of interest has been declared by the authors.

\section{Funding}




\section{Authors' contributions}

JL $R$ and $D Y Z$ conceived the study. JL R, $Y Z$ and $M L$ designed the questionnaire. $Y L$ and $R D X$ collected data. JL R and $Y L$ analyzed data. JL R drafted the manuscript. All authors are significantly involved in the study preparation and conduct. And all authors have read and approved the manuscript.

\section{Acknowledgements}

The authors would like to thank the members of the research team for their support.

\section{References}

1. Kreps S,Prasad S,Brownstein JS, Hswen Y, Garibaldi BT, Zhang B, Kriner DL. Factors Associated With US Adults' Likelihood of Accepting COVID-19 Vaccination. JAMA Netw Open 2020 Oct 1;3(10):e2025594. doi: 10.1001/jamanetworkopen.2020.25594.

2. Organization WHO. Ten threats to global health in 2019. https://www.who.int/vietnam/news/featurestories/detail/ten-threats-to-global-health-in-2019 (2019, accessed April 7, 2021).

3. MacDonald NE; SAGE Working Group on Vaccine Hesitancy. Vaccine hesitancy: Definition, scope and determinants. Vaccine 2015 Aug 14;33(34):4161-4164. doi: 10.1016/j.vaccine.2015.04.036.

4. Larson HJ, de Figueiredo A, Xiahong Z, et al. The State of Vaccine Confidence 2016: Global Insights Through a 67-Country Survey. EBioMedicine 2016; 12: 295-301. doi: 10.1016/j.ebiom.2016.08.042.

5. Kwok KO, Li K-K, Wei WI, et al. Editor's Choice: Influenza vaccine uptake, COVID-19 vaccination intention and vaccine hesitancy among nurses: A survey. Int J Nurs Stud 2021; 114: 103854. doi: 10.1016/j.ijnurstu.2020.103854.

6. 6..Taber KS. The Use of Cronbach's Alpha When Developing and Reporting Research Instruments in Science Education. Research in Science Education 2018; 48: 1273-1296. doi: 10.1007/s11165-0169602-2.

7. Giambi C, Fabiani $M$, Dancona F, et al. Parental vaccine hesitancyin Italy: results from a national survey. Vaccine 2018; 36(6): 779-787. doi: 10.1016/j.vaccine.2017.12.074.

8. Alabbad AA, Alsaad AK, Shaalan MA, et al. Prevalence of influenza vaccine hesitancy at a tertiary care hospital in Riyadh, Saudi Arabia. J Infect Public Health 2018;11(4):491-499.doi: 10.1016/j.jiph.2017.09.002.

9. Mengke Yu, Jiang Wu, Min Lv, et al. A review of social factors influencing vaccination. Chinese Journal of Vaccines and Immunization 2019; 25(03): 340344. https://kns.cnki.net/kcms/detail/detail.aspx?

FileName=ZGJM201903024\&DbName=DKFX2019 
10. Wang J, Jing R, Lai X, et al. Acceptance of COVID-19 Vaccination during the COVID-19 Pandemic in China. Vaccines (Basel) 2020; 8. doi: 10.3390/vaccines8030482.

11. Zhou Y, Zhang J, Wu W, Liang M, Wu QS. Willingness to receive future COVID-19 vaccines following the COVID-19 epidemic in Shanghai, China. BMC Public Health 2021 Jun 9;21(1):1103. doi: 10.1186/s12889-021-11174-0.

12. Lazarus JV, Wyka K, Rauh L, et al. Hesitant or Not? The Association of Age, Gender, and Education with Potential Acceptance of a COVID-19 Vaccine: A Country-level Analysis. J Health Commun2020 Oct 2;25(10):799-807. doi: 10.1080/10810730.2020.1868630.

13. Moran KR and Del Valle SY. A Meta-Analysis of the Association between Gender and Protective Behaviors in Response to Respiratory Epidemics and Pandemics. PLoS One 2016; 11: e0164541. doi: 10.1371/journal.pone.0164541.

14. Zintel S, Flock C, Arbogast AL, et al. Gender Differences in the Intention to Get Vaccinated against COVID-19 - a Systematic Review and Meta-Analysis (March 18, 2021). Available at SSRN: https://ssrn.com/abstract=3803323 or http://dx.doi.org/10.2139/ssrn.3803323.

15. Kreps S, Prasad S, Brownstein JS, et al. Factors Associated With US Adults' Likelihood of Accepting COVID-19 Vaccination. JAMA Netw Open 2020; 3: e2025594. doi: 10.1001/jamanetworkopen.2020.25594.

16. Pogue K, Jensen JL, Stancil CK, et al. Influences on Attitudes Regarding Potential COVID-19 Vaccination in the United States. Vaccines (Basel) 2020; 8. doi: 10.3390/vaccines8040582.

17. Wang K, Wong ELY, Ho KF, et al. Intention of nurses to accept coronavirus disease 2019 vaccination and change of intention to accept seasonal influenza vaccination during the coronavirus disease 2019 pandemic: A cross-sectional survey. Vaccine 2020; 38: 7049-7056. doi:

10.1016/j.vaccine.2020.09.021.

18. Lucia VC, Kelekar A and Afonso NM. COVID-19 vaccine hesitancy among medical students. J Public Health (Oxf) 2020. doi: 10.1093/pubmed/fdaa230.

19. Wagner AL, Huang Z, Ren J.et al.Vaccine Hesitancy and Concerns About Vaccine Safety and Effectiveness in Shanghai,China. Am J Prev Med. 2021 Jan;60(1 Suppl 1):S77-S86. doi: 10.1016/j.amepre.2020.09.003.

20. Shaw J, Stewart T, Anderson KB, et al. Assessment of U.S. health care personnel (HCP) attitudes towards COVID-19 vaccination in a large university health care system. Clin Infect Dis 2021. doi: 10.1093/cid/ciab054.

21. Harapan H, Wagner AL, Yufika A, et al. Acceptance of a COVID-19 Vaccine in Southeast Asia: A Cross-Sectional Study in Indonesia. Front Public Health 2020; 8: 381. doi: 10.3389/fpubh.2020.00381.

22. Leng A, Maitland E, Wang S, et al. Individual preferences for COVID-19 vaccination in China. Vaccine 2021; 39: 247-254. doi: 10.1016/j.vaccine.2020.12.009.

\section{Tables}


Due to technical limitations, tables are only available as a download in the Supplemental Files section.

\section{Supplementary Files}

This is a list of supplementary files associated with this preprint. Click to download.

- Tables.doc 